\title{
A VARIATIONAL PRINCIPLE AND AN ALGORITHM FOR LIMIT ANALYSIS OF BEAMS AND PLATES
}

\author{
Wei H. YANG \\ The University of Michigan, Ann Arbor, MI 48109, U.S.A.
}

\begin{abstract}
A variational principle for limit analysis of beams and plates is developed from a yield function based on the Frobenius matrix norm. The formulation produced a pair of maximization and minimization problems with a duality relation between them. Exact solutions of two simple problems are presented as verification to the validity of the new variational principle. An iterative algorithm is constructed to solve the minimization problem. The algorithm, tested successfully on the two example problems, is intended for beams and plates with general loading and boundary conditions and shapes.
\end{abstract}

\section{Introduction}

Plasticity as a model for mechanics of a class of materials presents some unusual mathematical difficulties that require special treatment. To correctly represent the slip-yield phenomenon of the crystalline structure, the field functions like the displacement and strain should be allowed discontinuous behavior. The lack of a one to one constitutive relation requires inequality or incremental representations. The differential geometry of large deformation further introduces unpleasant nonlinearities. Great efforts have been made to overcome these difficulties, leading to some large scale incremental computer programs $[1,2]$.

Limit analysis provides an alternative approach. It avoids the tedium of the incremental analysis and attains the limit solution directly. Earlier development of limit analysis was based on a set of overly restrictive assumptions such as rigidity and perfect plasticity [3]. The lack of progress in methodology many years after the limit analysis theorems also discourages its application to practical engineering problems. Limit analysis should be interpreted as the asymptotic analysis for elastoplasticity [4]. The mathematical structure of a variational principle which could be applied to limit analysis was studied as early as 1936 [5] without a physical context. Of course, it was unnoticed by the researchers in mechanics. According to the author of [5], the mathematical community did not pay much attention to it either. The variational inequality [6] is now an important chapter in nonlinear analysis. Its application to plasticity $[7,8]$ interests both mathematicians and engineers.

Limit analysis requires less input information. The constitutive relation is modeled by an inequality. This relaxed demand is quite welcome by the engineers since the stress-strain equation for many materials are either unavailable or uncertain. The output of the limit analysis provides crucial information in terms of the collapse load and collapse mode which engineers sought. On the other hand, the incremental analysis requires much more input and computes so much details some of which are hardly ever used in practice.

The variational principle developed here is based on a special yield function of the moment matrix, 


$$
f(M)=\sqrt{M_{x x}^{2}+2 M_{x y}^{2}+M_{y y}^{2}}-M_{0} \leqslant 0
$$

where $M_{x x}, M_{y y}$ are the bending moment components, $M_{x y}$ is the twisting moment and $M_{0}$ is a material constant. $f(M)=0$ describes the yield surface of a perfectly plastic plate or an envelope surface enclosing all possible states of moments. The plate is assumed stiff before collapse so small deflection is assumed up to the impending failure.

In the classical analysis of differential equations, we require not only that the solutions satisfy the conditions prescribed on the boundary but they approach these conditions smoothly. In the variational principle in this paper, we allow certain discontinuities of the functions. They still satisfy the boundary conditions but may approach them with a jump. For example, a plate may be clamped on a boundary therefore the slope there must be zero. The plate is allowed to approach such a boundary with a finite slope, then jump to zero slope at the boundary. This is physically quite admissible as plates and beams kink. The discontinuity of slope is also allowed in the interior as the minimization procedure dictates. The second derivatives of the deflection function $w(x, y)$ at these discontinuities are interpreted as a set of measures [9] whose integration is a jump.

The final formulation is in the form of minimizing an integral functional of the curvature matrix

$$
\nabla \nabla w=\left[\begin{array}{cc}
\partial^{2} w / \partial x^{2} & \partial^{2} w / \partial x \partial y \\
\partial^{2} w / \partial x \partial y & \partial^{2} w / \partial y^{2}
\end{array}\right]
$$

which is also called the Hessian matrix of the deflection $w(x, y)$.

Two simple examples are presented with their exact solutions. An algorithm is given for more complex problems. Test of convergence of the algorithm on the two cxample problems are shown.

\section{Formulation}

The $2 \times 2$ moment matrix

$$
M(x, y)=\left[\begin{array}{ll}
M_{x x} & M_{x y} \\
M_{x y} & M_{y y}
\end{array}\right]
$$

represents the state of moment at a point in a plate with domain $D$. Those functions $M$ which satisfy the equilibrium equation [10]

$$
\nabla \cdot(\nabla \cdot M)=q_{0} \varphi(x, y)
$$

and the limit criterion (1) are called the lower bound solutions, where $q_{0}$ is the load parameter and $\varphi(x, y)$ is a given distribution function. We seek the maximum possible $q_{0}$ without violating (1) and (4). This can be formally stated as a variational problem with constraints that include inequalities, 


$$
\begin{array}{cl}
\max & q_{0}, \\
\text { s.t. } & \nabla \cdot(\nabla \cdot M)=q_{0} \varphi, \\
& f(M)=\|M\|_{\mathrm{F}}-M_{0} \leqslant 0,
\end{array}
$$

Boundary Conditions on $M$,

where $\|M\|_{F}$ is the Frobenius norm of a matrix defined by the expression in (1). The above stated problem is named primal problem. Using the variational procedure, one can derive the dual problem for a given primal problem. We have such a paired formulation called the potential energy and the complementary energy in the theory of elasticity. The dual problem of (5) can be derived in the same manner as that in [10]. We shall omit the derivation here and only state the dual problem,

$$
\begin{array}{ll}
\min & M_{0} \iint_{D}\|\nabla \nabla w\|_{F} \mathrm{~d} A=q^{*}, \\
\text { s.t. } & \iint_{D} w \varphi \mathrm{d} A=1,
\end{array}
$$

Boundary Conditions on $w$,

where $q^{*} \geqslant q_{0}$ is a property of the duality relation between (5) and (6), and $w$ is sought in the space of all kinematically admissible functions to minimize $q^{*}$. We shall denote this space by $W$. Functions in $W$ must be continuous. Their first derivatives may take finite jumps. Their second derivatives at the jumps are defined as integrable measures. When such jumps are present, the contribution of the distributions must be added to the integral. Thus the $q^{*}$ in $(6)$ should be replaced by

$$
q^{*}=\min M_{0}\left(\iint_{D}\|\nabla \nabla w\|_{\mathrm{F}} \mathrm{d} A+\int_{\Gamma}\|\partial w / \nabla n\| \mathrm{d} s\right)
$$

where $\Gamma$ is the union of all curves of discontinuities with normal direction $n$ and arc length direction $s$ including the boundary $\partial D$, the brackets [ ] denote the jump of the quantity it encloses. Often the line integral is not explicitly written. Then the interpretation of the area integral must include the contributions from the distributions as the integrals in $W$ imply.

Two simple example problems can be solved exactly to demonstrate the application of this new variational principle. They are given in the next section.

\section{Simple examples}

\subsection{A clamped beam of length 2 a under a uniform load $q_{0}$}

The lower bound formulation (5) becomes one dimensional,

$$
\begin{aligned}
\max & q_{0}, \\
\text { s.t. } & M^{\prime \prime}=q_{0}, \quad-a \leqslant x \leqslant a, \quad|M|-M_{0} \leqslant 0,
\end{aligned}
$$


where ( $)^{\prime}$ denotes derivative with respect to $x$, and no boundary condition on $M$ is prescribed. For this simple problem the equilibrium equation can be integrated easily to give

$$
M(x)=\frac{1}{2} q_{0} x^{2}+C_{1} x+C_{2}
$$

where $C_{1}$ and $C_{2}$ are constants. Since $M(x)$ must be even by symmetry, $C_{1}=0$. The maxima and the minimum of $M$ in the interval $[-a, a]$ are $M(-a)=M(a)=\frac{1}{2} q_{0} a^{2}+C_{2}$ and $M(0)=C_{2}$, respectively. If we let $C_{2}=-M_{0}$ and $\frac{1}{2} q_{0} a^{2}+C_{2}=M_{0}$, then $q_{0}$ attains maximum

$$
q_{0}=4 M_{0} / a^{2} .
$$

The dual problem (6) has the form

$$
\begin{array}{ll}
\min & M_{0}\left(\int_{-a}^{a}\left|w^{\prime \prime}\right| \mathrm{d} x+\left|w^{\prime}(-a)\right|+\left|w^{\prime}(a)\right|\right)=q^{*}, \\
\text { s.t. } & \int_{-a}^{a} w \mathrm{~d} x=1, \quad w(-a)=w(a)=0, \quad w^{\prime}(-a)=w^{\prime}(a)=0 .
\end{array}
$$

Since we do not know the locations of the possible discontinuities in the interior, we shall compute them as they come.

One function,

$$
w(x)=(1-|x| / a)-a \leqslant x \leqslant a,
$$

satisfies all constraints and gives

$$
q^{*}=4 M_{0} / a^{2} .
$$

The fact, $q^{*} \geqslant q_{0}=4 M_{0} / a^{2}$ suggests that (13) is the true minimum. We do not claim however that $w(x)$ in (12) is unique. For this problem, $w(x)$ is unique. But for more complex limit analysis problems, many collapse modes may be associated to the same collapse load. The optimality $q^{*}$ is always unique.

\subsection{A simply supported circular plate of radius a with a concentrated load $P$ at its center}

The primal problem (5) is usually more difficult to solve than the dual problem (6). We shall present only the exact dual solution $w(r)$ for this circular plate.

By polar symmetry (6) reduces to

$$
\begin{aligned}
& \min 2 \pi \int_{0}^{1} \sqrt{r^{2} w_{r r}^{2}+w_{r}^{2}} \mathrm{~d} r=P^{*} / M_{0}, \\
& \text { s.t. } \quad w(0)=1, \quad w(1)=0 .
\end{aligned}
$$

Here we have normalized the variables and $P^{*} \geqslant P$. ( ) r denotes derivative with respect to $r$. 
Using the standard operations in calculus of variations one can derive the Euler's equation,

$$
\left(r^{2} w_{r} / S\right)_{r}-w_{r} / S=C, \quad w(0)=1, \quad w(1)=0
$$

where $S=\left(r^{2} w_{r}^{2}+w_{r}^{2}\right)^{1 / 2}$ and $C$ is an arbitrary constant. This third order ordinary differential equation requires one more boundary condition. In the case of linear analysis, we use the regularity condition $w_{r}(0)=0$. For functions in $W$ such a regularity condition cannot be taken for granted. The values of $w_{r}(0)$ and $C$ are determined by the minimization procedure. The exact solution for this problem happened to be simple. One can easily verify that

$$
w(r)=1-r
$$

satisfies (15) when $C=-1$ is chosen. The minimum value $P^{*} / M_{0}$ is $2 \pi$. This collapse mode agrees with the classical limit analysis solution [11]. The circular plates with other loading and boundary conditions do not assume the cone as the collapse mode. Exact numerical solutions of circular plates under various loading and boundary conditions are presented in [12].

The two simple examples only serve to verify some facts of the variational formulations (5) and (6). In Section 4 we give an iterative algorithm for numerical solutions of more general problems.

\section{An algorithm}

In the two examples we simply tried and succeeded using simple functions in $W$ to satisfy the variational principle. In general the exact solutions can only be approached iteratively and numerically. In this section an iterative algorithm is suggested and is applied to approach the known exact solutions of the examples.

We restrict the discussion for plates with either simply supported or clamped boundaries $\partial D$ of a general domain $D$. Other boundary conditions can be easily implemented. The loading on the plate is specified by a distribution function $\varphi(x, y)$ in $W$. The iterative scheme is constructed by rewriting (6) in the form

$$
\begin{array}{ll}
\min & \iint_{D} \frac{\left(w_{x x}^{2}+2 w_{x y}^{2}+w_{y y}^{2}\right)_{k}}{\left(w_{x x}^{2}+2 w_{x y}^{2}+w_{y y}^{2}\right)_{k-1}^{1 / 2}+\varepsilon} \mathrm{d} A+\oint_{\partial D}\left|\left[\frac{\partial w}{\partial n}\right]\right|_{k} \mathrm{~d} s=q_{k}^{*} / M_{0}, \\
\text { s.t. } & \iint_{D}(w)_{k} \varphi \mathrm{d} A=1, \\
& (w)_{k}=0 \text { on } \partial D, \quad\left(w_{n}\right)_{k}=0 \text { on } \partial D, \\
& k=1,2, \ldots,
\end{array}
$$

where the subscripts $x, y, n$ denote partial derivatives, $k$ denotes iteration index, $\varepsilon>0$ is a small parameter to protect from division by zero and $(w)_{0}$ is an assumed initial iterate. The line integral and the boundary condition $\left(w_{n}\right)_{k}=0$ are removed for the simply supported plates. 
The square root in the denominator is now a known function which serves as a temporary measure of local curvature. Its inverse is a weighting function for the quadratic functional. Therefore, small curvature weights more than large curvature. The minimization at each iteration will drive the regions with small curvature to further reduce their curvature. It is a distinct possibility that curvature of some region will be driven to zero. This is not surprising since limit solutions of plates are known to include rigid body deflection for part of the plate. The parameer $\varepsilon$ protects computation from such a possibility. The Richardson's extrapolation [13] can be used to obtain the solution for $\varepsilon=0$.

Since the functional in (17) is quadratic we can use the standard finite element method $|14|$ at each iterative step. The problem is similar to an elastic plate with variable thickness. The finite dimensional problem has the form

$$
\begin{array}{ll}
\min & \boldsymbol{w}_{k}^{\mathrm{t}} A\left(\boldsymbol{w}_{k-1}\right) \boldsymbol{w}_{k} \\
\text { s.t. } & \boldsymbol{c}^{\mathrm{t}} \boldsymbol{w}_{k}=1
\end{array} \quad k=1,2, \ldots
$$

where $w_{k}$ is the vector representation of $w_{k}(x, y), A$ is a symmetric positive definite matrix whose elements are functions of $w_{k-1}(x, y), c$ is a constant vector derived from the constraint integral.

At each iterative step $k$ we treat $A$ as a constant matrix and solve the quadratic programming problem,

$$
\min w^{t} A w-2 \lambda\left(c^{t} w-1\right)
$$

where $\lambda$ is the Lagrangian multiplier. The solution of (19) is

$$
\lambda=1 / c^{\mathrm{t}} v . \quad w=\lambda v . \quad w^{\mathrm{t}} A w=\lambda
$$

where $v$ is the solution of the sparse linear system $A v=c$. Using (20) we update the matrix $A$ and proceed to the next iteration until $\left\|\boldsymbol{w}_{k+1}-\boldsymbol{w}_{k}\right\|<\delta$, an error tolerance criterion.

Unlike the incremental method where the stiffness matrix becomes singular or ill-conditioned as we approach the limit solution, the matrix $A$ in the quadratic programming problem (19) is always well conditioned. Also unlike the incremental method, the error of the iterative solution is not accumulative.

We make no attempt to prove the convergence of this iterative algorithm. To test the convergence, the solutions of the two example problems in the previous section are computed using the iterative method. The Hermitian spline is used as the basis functions for the tinite elements. Forty elements are used for the normalized beam domain $[-1,1]$, and the plate domain [0.1], Hence the approximate solutions are relatively smooth. The initial and final iterations for the beam and the circular plate are shown in Fig. 1 and Fig. 2, respectively. The initially smooth iterates are driven by the algorithm to approach the kinks in the beam and the cone of the plate.

Minimization of a square root functional arises also in other fields. The most well known is the minimal surface problem 


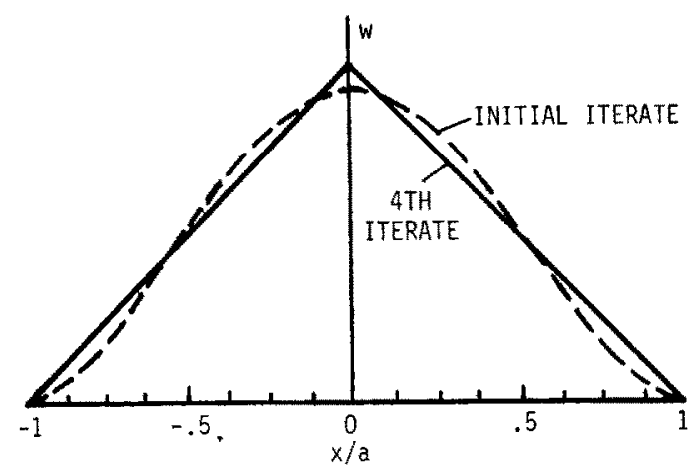

Fig. 1. Iterative solutions for a clamped beam under uniform load.

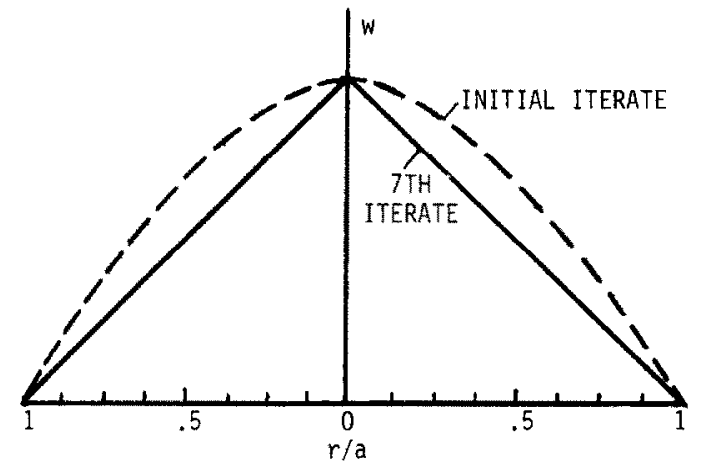

Fig. 2. Iterative solutions for a simply-supported circular plate under a centered force.

$$
\begin{aligned}
& \min \iint_{D} \sqrt{1+w_{x}^{2}+w_{y}^{2}} \mathrm{~d} A, \\
& \text { s.t. } w=g \text { on } \partial D,
\end{aligned}
$$

where subscripts $x, y$ denote partial derivatives and $g$ is a given function on the boundary $\partial D$. This problem has a wide interest shown by the large amount of literature $[15,16,17]$. We are following the other developments to further the advances in plasticity.

\section{Final remarks}

Although the upper and lower bound theorems are well known, the variational approach to limit analysis is relatively recent. The variational principles (5) and (6) involving the Frobenius matrix norm as a yield function for plates is new.

The two simple examples provide some credibility to the new formulation although the Frobenius norm as a good yield function for plates requires additional study both theoretically and experimentally. The yield criterion given in (1) is more conservative than the Johanson's maximum bending moment yield criterion [18]

$$
\|M\|_{2}-M_{0} \leqslant 0
$$

which predicts higher collapse loads than observed. It is expected that the solutions obtained from the new variational principle will be more realistic.

The iterative algorithm is simple and is adaptive to the existing finite element codes for variable thickness plates and the standard sparse matrix solution routines. Solutions to several boundary value problems are now being computed to provide data for an experimental project.

\section{References}

[1] H.A. Balmer, J.St. Doltsinis and M. Konig, Elasto-plastic and creep analysis with the ASKA Program System. Comp. Meths. Appl. Mech. Engrg. 3 (1974) 87-104. 
[2] T.G. Bulter and D. Michel, NASTRAN: a summary of the functions and capacities of the NASA structural analysis computer system, Scientific and Technical Information Office, NASA SP-60, 1971.

[3] A. Nádai, Plasticity (McGraw-Hill, New York, 1931).

[4] E.H. Lee, R.L. Mallett and W.H. Yang, Stress and deformation analysis of the metal extrusion process, Comput. Meths. Appl. Mech. Engrg. 10 (1977) 339-353.

[5] L. Cesari, Sulle funzioni a variazione limitata, Ann. R. Scuola Norm. Sup. Pisa. Sc. fis. met. II Ser. 5 (1936) 293-313.

[6] J.L. Lions and G. Stampacchia, Variational inequalities, Comm. Pure Appl. Math. 20 (1967) 493-519.

[7] R. Teman and G. Strang, Duality and relaxation in the variational problems of plasticity, J. Mecanique 19(3) (1980) 493-521.

[8] W.H. Yang, Pipe flow of plastic materials, Trans. ASME J. Appl. Mech. 47 (1980) 496-498.

[9] R.G. Bartle, The Elements of Integration (Wiley, New York, 1966).

[10] W.H. Yang, Minimization approach to limit solutions of plates, Comput. Meths. Appl. Mech. Engrg. 28(3) (1981) 265-274.

[11] E.H. Mansfield. Studies in collapse analysis of rigid-plastic plates with a square yield diagram. Proc. Roy. Soc. London Ser. A 241 (1957) 311-338.

[12] W.H. Yang, Exact solutions for limit analysis of circular plates with various loading and boundary conditions, AM Rept., The University of Michigan, MI, 1981.

[13] G. Dahlquist and A. Bjorck, Numerical Methods (Prentice-Hall, Englewood Cliffs, NJ, 1974).

[14] H.C. Martin and G. Carey, Introduction to Finite Element Analysis (McGraw-Hill, New York, 1973).

[15] P. Concus, Numerical solution of minimal surface equation, Math. Comput. 21 (1967) 340-350.

[16] M. Hinata, M. Shimasaki and T. Kitono, Numerical solution of Plateau's problem by a finite element method. Math. Comput. 28 (1974) 45-60.

[17] W.I.. Wilson, Jr., On discrete Dirichlet and Plateu problems, Numer. Math. 3 (1961) 359-373.

[18] K.W. Johanson, Congress Internat. Assoc. Br. Struct. Engrg. 1 (1932) 277-294. 